\title{
Outcomes in children undergoing posterior fossa decompression and duraplasty with and without tonsillar reduction for Chiari malformation type I and syringomyelia: a pilot prospective multicenter cohort study
}

\author{
*Joyce Koueik, MD, MS, ${ }^{1}$ Carolina Sandoval-Garcia, MD, ${ }^{1}$ John R. W. Kestle, MD, ${ }^{2}$ \\ Brandon G. Rocque, MD, MS, ${ }^{3}$ David M. Frim, MD, PhD, ${ }^{4}$ Gerald A. Grant, MD, ${ }^{5}$ \\ Robert F. Keating, MD, ${ }^{6}$ Carrie R. Muh, MD, ${ }^{7}$ W. Jerry Oakes, MD, ${ }^{3}$ lan F. Pollack, MD, ${ }^{8}$ \\ Nathan R. Selden, MD, PhD, ${ }^{9}$ R. Shane Tubbs, PhD, PA-C, ${ }^{3}$ Gerald F. Tuite, MD, ${ }^{10}$ \\ Benjamin Warf, MD, ${ }^{11}$ Victoria Rajamanickam, MS, ${ }^{12}$ Aimee Teo Broman, MA, ${ }^{12}$ \\ Victor Haughton, MD, ${ }^{13}$ Susan Rebsamen, MD, ${ }^{13}$ Timothy M. George, MD, ${ }^{14}$ and \\ Bermans J. Iskandar, MD'1
}

${ }^{1}$ Department of Neurological Surgery, University of Wisconsin, Madison, Wisconsin; ${ }^{2}$ Department of Neurosurgery, University of Utah, Salt Lake City, Utah; ${ }^{3}$ Department of Neurosurgery, Children's of Alabama, Birmingham, Alabama; ${ }^{4}$ Section of Neurosurgery, Department of Surgery, University of Chicago, Chicago, Illinois; ${ }^{5}$ Department of Pediatric Neurosurgery, Stanford Health Care, Palo Alto, California; ${ }^{6}$ Department of Neurosurgery, Children's National Health System, Washington, DC; ${ }^{7}$ Department of Neurosurgery, Duke University Medical Center, Durham, North Carolina; ${ }^{8}$ Department of Neurosurgery, UPMC Children's Hospital of Pittsburgh, Pennsylvania; ' Department of Neurological Surgery, Oregon Health and Science University, Portland, Oregon; ${ }^{10}$ Department of Neurosurgery, Johns Hopkins All Children's Hospital, Tampa, Florida; ${ }^{11}$ Department of Neurosurgery, Boston Children's Hospital, Boston, Massachusetts; Departments of ${ }^{12}$ Biostatistics and Medical Informatics and ${ }^{13}$ Radiology, University of Wisconsin-Madison, Wisconsin; and ${ }^{14}$ Department of Neurosurgery, Dell Medical School, Austin, Texas

OBJECTIVE Despite significant advances in diagnostic and surgical techniques, the surgical management of Chiari malformation type I (CM-I) with associated syringomyelia remains controversial, and the type of surgery performed is surgeon dependent. This study's goal was to determine the feasibility of a prospective, multicenter, cohort study for CM-I/syringomyelia patients and to provide pilot data that compare posterior fossa decompression and duraplasty (PFDD) with and without tonsillar reduction.

METHODS Participating centers prospectively enrolled children suffering from both $\mathrm{CM}-\mathrm{I}$ and syringomyelia who were scheduled to undergo surgical decompression. Clinical data were entered into a database preoperatively and at 1-2 weeks, 3-6 months, and 1 year postoperatively. MR images were evaluated by 3 independent, blinded teams of neurosurgeons and neuroradiologists. The primary endpoint was improvement or resolution of the syrinx.

RESULTS Eight clinical sites were chosen based on the results of a published questionnaire intended to remove geographic and surgeon bias. Data from 68 patients were analyzed after exclusions, and complete clinical and imaging records were obtained for 55 and 58 individuals, respectively. There was strong agreement among the 3 radiology teams, and there was no difference in patient demographics among sites, surgeons, or surgery types. Tonsillar reduction was not associated with $>50 \%$ syrinx improvement $(R R=1.22, p=0.39)$ or any syrinx improvement $(R R=1.00, p=0.99)$. There were no surgical complications.

CONCLUSIONS This study demonstrated the feasibility of a prospective, multicenter surgical trial in CM-l/syringomyelia

ABBREVIATIONS ASAP = American Syringomyelia and Chiari Alliance Project; $\mathrm{CM}-\mathrm{I}=$ Chiari malformation type I; $\mathrm{PFD}=$ posterior fossa decompression; $\mathrm{PFDD}=\mathrm{PFD}$ and duraplasty; PFDD-T = PFDD with tonsillar reduction.

SUBMITTED April 10, 2019. ACCEPTED August 1, 2019

INCLUDE WHEN CITING Published online October 18, 2019; DOI: 10.3171/2019.8.PEDS19154.

* J.K. and C.S.G share first authorship, and T.M.G. and B.J.I. share senior authorship of this work. 
and provides pilot data indicating no discernible difference in 1-year outcomes between PFDD with and without tonsillar reduction, with power calculations for larger future studies. In addition, the study revealed important technical factors to consider when setting up future trials. The long-term sequelae of tonsillar reduction have not been addressed and would be an important consideration in future investigations.

https://thejns.org/doi/abs/10.3171/2019.8.PEDS19154

KEYWORDS Chiari malformation decompression; tonsillar resection; syrinx improvement; surgical outcomes

$\mathrm{C}$ HIARI malformation type I (CM-I) consists of a caudal position of the cerebellar tonsils in the upper cervical spinal canal. The accessibility of MRI has led to an increase in CM-I diagnosis, which is detected at an average age of $24.9 \pm 15.8$ years. ${ }^{19}$ While acquired tonsillar ectopia has been reported as a consequence of hydrocephalus, trauma, brain tumors, ${ }^{22}$ and other etiologies (e.g., lumboperitoneal shunting), ${ }^{29}$ true CM-I is thought to be congenital.

Syringomyelia is a fluid-filled cavity within the spinal cord that occurs secondary to a variety of etiologies, including tumor, trauma, tethered cord, arachnoiditis, or, as in the present study, CM-I. While surgical series of CM-I decompression have reported a $65 \%-80 \%$ rate of co-occurrence of syringomyelia, ${ }^{8,19,26}$ imaging and epidemiological CM-I studies have shown only a $23 \%$ rate of co-occurrence. ${ }^{24}$

If warranted, the surgical treatment for CM-I consists of enlargement of the posterior cranial fossa. The decision to treat CM-I surgically is based on the severity and progression of symptoms and signs, in conjunction with MRI and CSF flow findings. Most, though not all, pediatric neurosurgeons agree that the presence of significant syringomyelia in association with CM-I is an indication for posterior fossa decompression (PFD), even in the absence of symptoms..$^{21,25}$ However, there is major controversy about the surgical technique that is used. While some advocate bone-only craniocervical decompression (typically craniectomy and $\mathrm{C} 1$ posterior arch removal), others recommend bone decompression and duraplasty, and still others recommend bone decompression and duraplasty with tonsillar reduction. ${ }^{21}$ While it is agreed generally that avoiding a dural opening leads to a lower complication rate, a rigorous comparison of the outcomes of various surgical techniques is unavailable, as most studies to date have examined small, single-center, retrospective cohorts. ${ }^{5,7,17}$

The goals of this pilot trial were to demonstrate the feasibility of conducting a prospective, multicenter, surgical cohort comparison study of CM-I and syringomyelia patients by establishing a cooperative group of centers to work toward the goal of a future randomized trial, allow the sample size and power for such a trial to be estimated, and provide 1-year outcome data that compare two standard CM-I decompression procedures: craniectomy, C1 posterior arch removal, and duraplasty without tonsillar reduction (PFDD) and craniectomy, $\mathrm{C} 1$ posterior arch removal, and duraplasty with tonsillar reduction (PFDD-T).

\section{Methods}

\section{Trial Design}

This was a multicenter, prospective, nonrandomized surgical trial for patients with CM-I and syringomyelia that compared the outcomes of PFDD with and without tonsillar resection/reduction. The primary hypothesis was that the two procedures were equivalent, with syrinx improvement as the primary outcome and symptomatic improvement as the secondary outcome.

The study concept and design originated from the following: 1) a survey of the membership of the American Syringomyelia and Chiari Alliance Project (ASAP), the largest patient-support network in North America, which indicated that the surgical approach used to decompress a CM-I is the most critical clinical question to answer (unpublished), and 2) a survey of the American Society of Pediatric Neurosurgeons, which revealed important trends and differences in treatment practices across the US and highlighted the relevance of establishing outcome comparisons across centers to make appropriate management recommendations. ${ }^{21}$

\section{Patient Recruitment and Clinical Data}

After obtaining IRB approval for the study from all participating centers, pediatric patients diagnosed with both CM-I and syringomyelia were recruited. Inclusion criteria included: children aged 0 to 17 years; a CM-I with tonsil position at least $5 \mathrm{~mm}$ below the foramen magnum; a syrinx with a width $>50 \%$ of the cross-sectional diameter of the spinal cord, and a length of at least 2 cervical and/or thoracic vertebral segments. Exclusion criteria included one or more of the following preexisting conditions: hydrocephalus; tumor; brain trauma; stroke; meningitis; abscess; other congenital anomalies of the brain or skull base; vertebral anomalies (i.e., Klippel-Feil, atlas assimilation, etc.); degenerative or demyelinating disease; CM-I without syringomyelia; syringomyelia with cerebellar tonsils in a normal position; fibromyalgia; chronic fatigue syndrome; and prior posterior fossa surgery. Patients unable to provide consent were also excluded. Standardized forms were used for patient demographics, radiological assessments, surgical details, and clinical follow-ups. Data collected included pre- and postoperative signs and symptoms, pre- and postoperative MR images of the brain and entire spine, details of the surgical intervention, and postoperative complications. The Office of Clinical Trials in the University of Wisconsin Institute for Clinical and Translational Research managed the study. De-identified, uniform prospective data were entered and stored in an electronic database. OnCore (Forte Research, Inc.) was used as the study and data management software. Financial reimbursement included $\$ 3000$ per center to cover IRB fees, and an additional $\$ 500$ per subject recruited.

\section{Surgeons and Centers}

While the study allowed individual surgeons to follow 
clinical care algorithms and decisions practiced at their institutions routinely, the centers were selected based on answers to the American Society of Pediatric Neurosurgeons survey, ${ }^{21}$ with 2 main goals: 1 ) to choose surgeons whose standard of practice after Chiari malformation decompression included obtaining pre- and postoperative brain and spinal MR images and clinical follow-up at 2-4 months and at 1-2 years postoperatively and 2) to ensure surgeon, center, and geographic diversity (Table 1). The trial was designed to minimize bias by doing the following: 1) recruiting centers at which the primary investigators (with one exception added later) were not ASAP (funding agency) medical advisory board members and 2) having an independent management and data coordinating center (University of Wisconsin, primary investigator: B.J.I. and co-primary investigator T.M.G.), and pre-study statistical consultant (J.R.W.K.), none of whom participated in subject recruitment. No change or modification in practice was required, as all surgeons followed their own routine diagnostic, surgical, and postoperative care. The goal was to accrue $8-12$ patients per center.

\section{Surgical Technique}

Originally, the study included 3 procedures: PFD with tonsillar reduction; PFD without tonsillar reduction, and bone-only PFD. The bone-only procedure was eliminated early in the study because of the dropping out of the 2 centers that perform the procedure routinely (before any subject accrual) and the remaining centers' lack of recruitment of patients undergoing the bone-only procedure ( 2 subjects, removed from analysis). At approximately the time the study began, only $7 \%$ of senior pediatric neurosurgeons surveyed performed bone-only decompressions routinely. ${ }^{21}$ As a result, all study patients underwent suboccipital craniectomy, $\mathrm{C} 1$ and occasionally $\mathrm{C} 2$ laminectomy, and duraplasty without manipulation of the cerebellar tonsils (PFDD) or PFDD with tonsillar reduction (PFDDT). Six centers performed both types of decompression, depending on the surgeon, and 2 performed tonsillar reduction in all patients accrued. Individually, all surgeons performed only one type of surgery routinely.

\section{Radiological Assessment}

The majority of patients underwent pre- and postoperative MRI of the brain and entire spine (with or without cine flow assessment). Those who were missing scans considered crucial for data analysis (i.e., brain and cervical spine MR images) were excluded from primary endpoint analysis (but included in secondary endpoint analysis if clinical data were available). De-identified MR images were collected and reviewed in the coordinating center. The postoperative MR images were obtained from 3 months to 1 year after surgery based on the routine practices of the various surgeons and centers.

The change in syrinx size was graded by 3 independent groups of evaluators as follows: grade 1: $>50 \%$ improvement; grade $2: \leq 50 \%$ improvement; and grade 3 , worsening or no change. None of the evaluators were involved in the study patients' clinical care. Evaluator team 1 consisted of 2 senior pediatric neurosurgeons who evaluated the images as a group; team 2 consisted of a senior neurosurgery resident and a senior neuroradiologists who evaluated the images as a group; and evaluator 3 was a senior neuroradiologist. All evaluators were blinded to patient identifiers and surgery group. Of course, it is impossible to blind the evaluators completely, as one theoretically might recognize a tonsil that has been surgically reduced in size.

\section{Outcomes Evaluation}

The primary outcome was designated as improvement or resolution of the syrinx, defined as $>50 \%$ improvement in length, maximal cross-sectional diameter, or both. The secondary outcome was designated as improvement in any clinical signs or symptoms.

\section{Data Quality}

An electronic data collection system, with a standard screening and data collection sheet, was used to ensure consistent data collection and reporting. A research coordinator reviewed the data at regular intervals for completeness and quality and to ensure protocol adherence.

\section{Statistical Analysis}

Patient demographics, hospital characteristics, and baseline symptoms were compared between surgery types and the primary endpoint of syrinx improvement using Fisher's exact test for categorical variables and the twosample t-test or Wilcoxon rank-sum test for continuous variables. To investigate the association between surgery type and syrinx improvement, we compared the "risk of improvement" between the two groups using relative risk, which is the proportion of patients who improved after PFDD-T divided by the proportion of those who improved after PFDD. To test the strength of association between improvement and the surgery groups, we used logistic models with a $\log$ link, which estimates the relative risk and confidence intervals using maximum likelihood. ${ }^{6}$ Outcomes of interest were $>50 \%$ syrinx improvement compared to no improvement or $\leq 50 \%$ syrinx improvement; and any syrinx improvement compared to no improvement. We used similar relative risk ratio estimation to determine if any association existed between secondary measures of symptom improvement, among those who experienced the symptom presurgery. Power calculations for future studies are based on the assumption that one of the two proportions is known, and we tested the null hypothesis that the second proportion is equal to the first. ${ }^{32}$

\section{Results}

\section{Accrual and Exclusions}

The study's original intent was to recruit 120 patients from 12 clinical sites over a 2-year period, with 8-12 patients per site, and 30-50 patients in each of the 3 surgical procedures (bone-only PFD, PFDD, and PFDD-T). Early in the planning phases and before any patient accrual, the bone-only PFD procedure was eliminated, as described in the Surgical Technique section. Accordingly, the study's goals were amended to 70-90 patients from 8 clinical sites. Final accruals were 75 patients in a 4-year- 


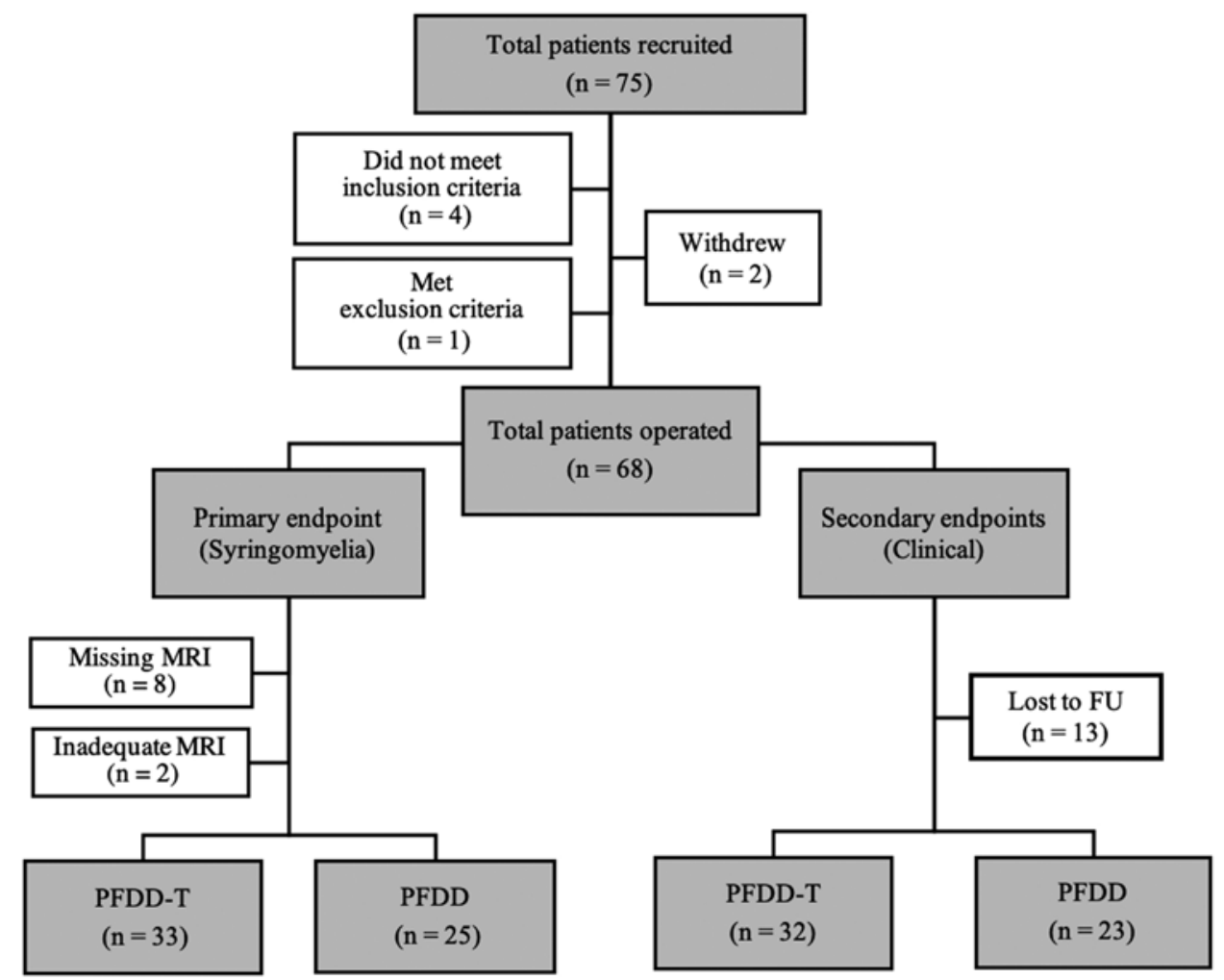

FIG. 1. Study inclusions and exclusions: This flowchart depicts all patients recruited into the study, including all inclusions and exclusions leading to the final group analyzed. FU = follow-up.

period, with an average of 8 individuals (range of 6-12) per center. Of the 75 patients who consented to enter the study originally, 4 were excluded for not meeting inclusion criteria (2 patients did not meet syrinx size criteria, and 2 patients had no duraplasty performed); 1 patient was excluded for meeting exclusion criteria (hydrocephalus); and 2 patients withdrew for personal reasons, leaving 68 patients for analysis.

\section{Data Completeness and Quality}

Preoperative image quality was deemed adequate (i.e., good visualization of brain and spinal cord, including the tonsils and syrinx) in $96.8 \%, 96.8 \%, 93.7 \%$, and $92.2 \%$ of the brain, cervical, thoracic, and lumbar MR images, respectively, while postoperative quality was deemed adequate in $96.8 \%, 93.7 \%, 82.8 \%$, and $75 \%$, respectively. Ten patients were eliminated from the primary endpoint analysis based on inadequate ( 2 cases) or missing ( 8 cases) MR images (Fig. 1). Those who were excluded from the primary endpoint analysis but had complete clinical data were included in secondary endpoint analysis.

\section{Patient Demographics}

Of the 68 patients studied, 37 were female (54.4\%) and 31 were male $(45.6 \%)$. Thirty-eight patients were white non-Hispanic (55.9\%), 15 were African American (22.1\%), and 15 were grouped as others $(22.1 \%)$, specifically 4 Hispanic, 3 Asian, 1 Native American/Alaskan Native, and 7 unknown. Patient ages ranged from 2 to 17 years, with a mean of 9.72 and a median of 9 years.

A total of 14 surgeons performed 68 surgical procedures, with an average of 4.8 procedures per surgeon (Table 1). Forty-two patients underwent PFDD-T (61.8\%) and 26 underwent PFDD (38.2\%). These constituted the two procedures in the study, and the two surgical groups' demographic information is shown in Table 2. There were no statistically significant differences in the populations' demographics related to type of surgery performed, except for study site and surgeon. No demographic variables were different between study groups $(\mathrm{p}>0.2)$, so none were included in the multivariate model. A two-sample t-test also showed no differences in baseline tonsillar position or syrinx size between the groups ( $p>0.37$ and 0.89 , respectively). All patients underwent expansile duraplasty using a graft. Autografts were used in $47.1 \%$ and allografts in $52.9 \%$.

\section{Follow-Up and Retention}

Postoperatively, 13 patients were lost to clinical followup by 1 year, and 10 had inadequate or incomplete MRI studies (Fig. 1). Postoperative visits occurred at the following intervals: first visit, 2-4 weeks; second visit, 3-6 months; and third visit, approximately 1 year. Sixty-six patients $(97 \%)$ were evaluated at the first postoperative visit, $63(92.6 \%)$ at the second, and $55(80.8 \%)$ at the third, such that clinical follow-up data were available for 55 patients at the end of the trial. 
TABLE 1. Participating centers and surgeon/patient enrollment

\begin{tabular}{lcc}
\hline \multicolumn{1}{c}{ Participating Centers } & $\begin{array}{c}\text { No. of } \\
\text { Patients }\end{array}$ & $\begin{array}{c}\text { No. of } \\
\text { Surgeons }\end{array}$ \\
\hline All Children's Hospital, St. Petersburg, FL & 9 & 2 \\
\hline Boston Children's Hospital, Boston, MA & 10 & 1 \\
\hline $\begin{array}{l}\text { Children's National Medical Center, Washington, } \\
\text { DC }\end{array}$ & 8 & 3 \\
\hline Duke University, Durham, NC & 8 & 2 \\
\hline $\begin{array}{l}\text { Oregon Health and Science University, Port- } \\
\quad \text { land, OR }\end{array}$ & 7 & 1 \\
\hline $\begin{array}{l}\text { Children's of Alabama, Birmingham, AL } \\
\text { University of Chicago, Chicago, IL }\end{array}$ & 12 & 1 \\
\hline University of Pittsburgh, Pittsburgh, PA & 6 & 1 \\
\hline
\end{tabular}

The numbers represent patients who underwent surgery after meeting inclusion criteria. See Fig. 1, which depicts the final numbers in each of the surgical groups, along with the various exclusions.

\section{Primary Outcome: Syrinx Improvement}

Of the 68 patients who met the inclusion criteria, 58 had pre- and postoperative MR images that were complete and were of adequate quality for evaluation. The other 10 were randomly distributed among 6 of the 8 centers. MR images were obtained at a mean of $5.5 \pm 3.6(\mathrm{SD})$ months and $4.9 \pm 2.4$ months in the PFDD and PFDD-T groups ( $\mathrm{p}=$ 0.98 [Wilcoxon rank-sum test]), respectively. The 3 groups of evaluators scored the images independently, and the weighted kappa analysis demonstrated strong interobserver agreements between all pairs of the 3 evaluator groups (kappa $=0.85-0.95$ : Table 3). Syrinx size decreased in $83 \%-88 \%$ of patients depending on the evaluator group (Table 4). Given the high agreement among the evaluators, we used the measures of improvement from evaluator 1 as the summary measure of all 3 evaluators.

The primary endpoint chosen during study planning was $>50 \%$ syrinx improvement. The proportion of patients who had $>50 \%$ improvement in syrinx size was $52 \%$ in the PFDD group and $63.6 \%$ in the PFDD-T group $(\mathrm{RR}=1.22, \mathrm{p}=0.39)$, with no correlation between outcome and timing of postoperative MRI. The proportion of patients who had any improvement (compared to no improvement) was $88 \%$ in both the PFDD and PFDD-T groups $(\mathrm{RR}=1.00, \mathrm{p}=0.99$; Table 5$)$.

\section{Secondary Outcome: Clinical Symptoms and Signs}

Of the 68 patients who met inclusion criteria, 55 had complete 1-year follow-up data, and 44 of these had presenting symptoms and signs, including the following: occipital headaches (28 patients [41.2\%]); frontal headaches (22 patients [32.4\%]); Valsalva maneuver-related symptoms (12 patients [17.6\%]); neck movement-related symptoms (8 patients [11.9\%]); oropharyngeal dysfunction, that is, swallowing difficulties, dysarthria, and/or apnea (10 patients $[14.7 \%]$ ); sensorimotor symptoms, that is, of the face, hand, arm, and leg (17 patients [25\%]), and cranial nerve symptoms, that is, blurry vision, diplopia, visual acuity, tongue atrophy/fasciculations, and nystagmus (17 patients [25\%]). Physical examination findings included
TABLE 2. Patient demographics

\begin{tabular}{|c|c|c|}
\hline \multirow[b]{2}{*}{ Variable } & \multicolumn{2}{|c|}{ Surgical Treatment } \\
\hline & PFDD & PFDD-T \\
\hline \multicolumn{3}{|l|}{ Sex, no. $(\%)$} \\
\hline Female & $14(54 \%)$ & $23(55 \%)$ \\
\hline Male & $12(46 \%)$ & $19(45 \%)$ \\
\hline \multicolumn{3}{|l|}{ Race/ethnicity, no. (\%) } \\
\hline White non-Hispanic & $17(65 \%)$ & $21(50 \%)$ \\
\hline Black/African American & $4(15 \%)$ & $11(26 \%)$ \\
\hline Other & $5(19 \%)$ & $10(24 \%)$ \\
\hline Age in yrs, median (range) & $10(3-17)$ & $8.5(2-17)$ \\
\hline \multicolumn{3}{|l|}{ Measurements on MRI } \\
\hline Tonsillar descent in $\mathrm{mm}$, mean $\pm \mathrm{SD}$ & $10.4 \pm 3.8$ & $11.6 \pm 2.9$ \\
\hline Syrinx length in $\mathrm{mm}$, mean $\pm \mathrm{SD}$ & $133.2 \pm 90.43$ & $134.6 \pm 86.25$ \\
\hline Ratio of syrinx/cord diameter & 0.72 & 0.68 \\
\hline
\end{tabular}

There were no statistically significant differences in any demographic between the surgical groups.

motor abnormalities (i.e., motor weakness in the upper and lower limbs, or hyperreflexia/Babinski sign in 11 patients $(16.2 \%)$; cerebellar dysfunction (i.e., ataxia, upperor lower-extremity coordination problems) in 12 patients (17.6\%); and papilledema in 2 patients (3\%). While specific symptoms and signs showed non-statistically significant differences between the two surgical groups (Table 6), $88.3 \%$ and $88.4 \%$ of patients exhibited improvement in at least one clinical category in the PFDD and PFDD-T groups, respectively.

\section{Complications}

There were no CSF leaks, pseudomeningoceles, wound problems, or neurological deficits. No patient underwent repeat surgery within the 1-year follow-up period.

\section{Protocol Variance}

Although all patients gave oral consent prior to surgery, 8 signed their consent in the first postoperative week. This was a protocol deviation reported to the IRB, which led to specific corrective measures described below, but the patients remained in the study.

\section{Discussion \\ General Conclusions}

This prospective multiinstitutional cohort pilot study demonstrates the feasibility of a multicenter surgical trial of CM-I associated with syringomyelia, establishes a collaborative group, and sets the stage for future large studies. The study results showed no advantage of tonsillar reduction at 1 year, with a distribution of presenting symptoms and signs consistent with that in the literature. ${ }^{11,19,27}$ When $>50 \%$ improvement in syrinx size was used as a primary outcome measure, there was no statistically significant difference between PFDD and PFDD-T. However, in this pilot study, our power to show that magnitude of difference was low. Longer follow-up and/or a larger study population would address this issue more conclusively. 
TABLE 3. Agreement among evaluators

\begin{tabular}{cc}
\hline Interobserver Agreement & Weighted Kappa \\
\hline Evaluator 1 vs evaluator 2 & 0.8591 \\
\hline Evaluator 1 vs evaluator 3 & 0.8578 \\
\hline Evaluator 2 vs evaluator 3 & 0.9513 \\
\hline
\end{tabular}

Weighted kappa analysis showed strong agreements among the blinded evaluators in their assessments of syrinx improvement. See Radiological Assessment section for a description of evaluators.

\section{Study Strengths and Weaknesses}

The present study has several strengths as follows: 1) while it was not powered to detect differences among center, surgeon, or geographic location, the methodology minimized study bias related to these parameters; and 2) it was prospective. Study weaknesses included: 1) a small sample size; 2) a short follow-up period; and 3) a lack of randomization. Because randomized surgical trials are costly and difficult to carry out for ethical/logistic reasons, a large multicenter prospective study or registry comes closest to providing sufficiently high-quality data to be clinically useful.

\section{Powering Future Studies}

If symptom improvement is to be considered the most important and clinically relevant endpoint, a power analysis would depend on the symptom chosen and the acknowledgment that the symptomatic state of this patient population is complex and inconsistent, often with symptoms of varied severity. Accordingly, large registries might be better suited than the typically smaller randomized protocols to study clinical endpoints.

Alternatively, based on our results, powering a study using the imaging primary endpoint of "any syrinx improvement" would not be feasible. Since PFDD and PFDD-T were virtually the same $(88.3 \%$ vs $88.4 \%$ exhibiting improvement in at least one clinical category [Table 6]), the sample size would be nearly infinite. A study with the primary endpoint of $>50 \%$ syrinx improvement maybe more doable. To power a future study with a similar difference in the proportion of patients to this study (52\% vs $64 \%$ ) with $>50 \%$ improvement, we would need approximately 265 patients per surgical group for an $80 \%$ power to detect a difference. Larger underlying effect sizes (e.g., a 50\% difference in outcome between the two groups) would reduce the number of study subjects needed and be more

TABLE 4. Primary endpoint of syrinx improvement

\begin{tabular}{cccc}
\hline Syrinx Improvement $(\mathrm{n}=58)$ & $\begin{array}{c}\text { Evaluator } \\
\text { Team 1 }\end{array}$ & $\begin{array}{c}\text { Evaluator } \\
\text { Team 2 }\end{array}$ & Evaluator 3 \\
\hline Improved & $51(88 \%)$ & $50(86 \%)$ & $48(83 \%)$ \\
\hline$>50 \%$ improvement & $34(59 \%)$ & $35(60 \%)$ & $37(64 \%)$ \\
\hline$\leq 50 \%$ improvement & $17(30 \%)$ & $15(26 \%)$ & $11(19 \%)$ \\
\hline No change/worse & $7(12 \%)$ & $8(14 \%)$ & $10(17 \%)$ \\
\hline
\end{tabular}

Postoperative syrinx improvement was consistently significant (above $82 \%$ ) among the 3 evaluators.
TABLE 5. Primary endpoint: syrinx improvement versus type of surgery based on evaluator team 1

\begin{tabular}{ccccc}
\hline \multirow{2}{*}{$\begin{array}{c}\text { Syrinx } \\
\text { Improvement }\end{array}$} & \begin{tabular}{c} 
Surgical Treatment \\
PFDD \\
\cline { 2 - 4 }$(\mathrm{n}=25)$
\end{tabular} & $\begin{array}{c}\text { PFDD-T } \\
(\mathrm{n}=33)\end{array}$ & $\begin{array}{c}\text { Risk Ratio } \\
(95 \% \mathrm{Cl})\end{array}$ & $\begin{array}{c}\mathrm{p} \\
\text { Value }\end{array}$ \\
\hline$>50 \%$ improvement & $13(52 \%)$ & $21(63.7 \%)$ & $1.22(0.78,1.93)$ & 0.39 \\
\hline Any improvement & $22(88 \%)$ & $29(88 \%)$ & $1.00(0.82,1.21)$ & 0.99 \\
\hline
\end{tabular}

PFDD-T was slightly more likely than PFDD to show syrinx improvement by $>$ $50 \%$ at 1 year after surgery, but the difference was not statistically significant. Syrinx improvement by any amount showed equivalence between the two surgery types. Note that the risk ratio indicated the probability of improvement.

persuasive in changing clinical practice (Table 7). In other words, if a larger study proves that tonsillar resection confers a $20 \%$ advantage over duraplasty alone, would that compel a traditional "duraplasty" neurosurgeon to start reducing tonsils? If not, would a $30 \%$ difference do so? Or a $50 \%$ difference? Furthermore, since syrinx improvement or resolution in response to successful PFD may not be discerned before 1 year postoperatively, ${ }^{14,30,33}$ this study does not address the possibility that longer followup might show smaller differences in outcome between surgery types.

\section{Challenges Encountered in Study Planning and Execution}

As a pilot study designed to provide feasibility for future large trials, one of our main goals was to identify potential challenges. To that end, we chose to describe the difficulties encountered in running the study in great detail and with full transparency. Since these problems affected small subsets of patients and were not confined to particular centers or study groups, they did not significantly alter study results.

\section{Patient Recruitment Problems}

Although the stringent inclusion criteria of syrinx size that we adopted led to a lower accrual of patients than expected, the most important issue in patient recruitment was the frequent loss or turnover of coordinators at the participating sites. Several strategies were adopted to remedy this problem: 1) We decreased the number of centers by eliminating those with significant challenges; 2) we initiated almost monthly communications with site coordinators and, when unavailable, contacted the local primary investigators; and 3) we provided a quarterly newsletter that reported progress and challenges. An additional option that might improve recruitment and coordinator dependability in future studies is increasing the reimbursement rate per patient.

\section{Data Entry Problems}

The principal problem with data entry was receipt of incomplete data sheets from the clinic visits, a problem that seemed related to the unavailability or poor retention of local coordinators. These omissions did not affect the outcome measures, as they rarely included the common symptoms and signs described in Table 6. Larger studies would provide more funding to ensure consistent local co- 
TABLE 6. Secondary endpoint: clinical improvement by the 1-year follow-up visit

\begin{tabular}{|c|c|c|c|c|c|c|}
\hline \multirow[b]{3}{*}{ Symptom } & \multicolumn{4}{|c|}{ Number of Patients (\%) } & \multirow[b]{3}{*}{$\begin{array}{c}\text { Risk Ratio } \\
(95 \% \mathrm{Cl})\end{array}$} & \multirow[b]{3}{*}{ p Value } \\
\hline & \multicolumn{2}{|c|}{ PFDD } & \multicolumn{2}{|c|}{ PFDD-T } & & \\
\hline & $\begin{array}{c}\text { Present at } \\
\text { Baseline }\end{array}$ & $\begin{array}{c}\text { Improvement } \\
\text { at } 1 \mathrm{Yr}\end{array}$ & $\begin{array}{c}\text { Present at } \\
\text { Baseline }\end{array}$ & $\begin{array}{c}\text { Improvement } \\
\text { at } 1 \mathrm{Yr}\end{array}$ & & \\
\hline Headache & 13 & $10(76.9 \%)$ & 23 & $21(91.3 \%)$ & $1.19(0.86,1.64)$ & 0.30 \\
\hline Valsalva/neck-related symptoms & 7 & $3(42.8 \%)$ & 10 & $9(90 \%)$ & $2.1(0.87,5.06)$ & 0.10 \\
\hline Cranial nerve & 11 & $7(63.63 \%)$ & 8 & $8(100 \%)$ & $1.57(-)$ & - \\
\hline Motor/sensory & 7 & $5(71.4 \%)$ & 9 & $7(77.7 \%)$ & $1.09(0.61,1.95)$ & 0.77 \\
\hline Cerebellar & 7 & $4(51.14 \%)$ & 5 & $5(100 \%)$ & $1.75(-)$ & - \\
\hline Any symptom/sign & 18 & $16(88.89 \%)$ & 26 & $23(88.46 \%)$ & $0.99(0.80,1.23)$ & 0.96 \\
\hline
\end{tabular}

$-=$ not calculated

This table shows the rates of clinical improvement by the 1-year follow-up visit. Note that individual patients often had multiple symptoms or signs, which explains the clinical outcome differences between the two surgical groups when based on "any symptom and sign" versus individual symptoms and signs. Confidence intervals and $p$ values were not calculated for cross-tables that had 0 observations in a cell.

ordinator availability. No additional issues with data entry were found.

\section{Protocol Variance}

The only protocol variance identified related to the delay in obtaining 8 patients' written consent. The IRB was notified of the deviation and discussions ensued with the local centers responsible to prevent further occurrences. In addition, the issue was highlighted in the newsletter to caution other centers about the problem.

\section{Patient Management Inconsistencies}

The study was designed to follow surgeons' own routine in the care of CM-I/syringomyelia patients, and management consistency was optimized by recruiting centers/ surgeons originally with similar pre- and postoperative routines-i.e., timing of clinic visits and MRI scans (determined with a survey). ${ }^{21}$ Despite these precautions, inconsistencies in management did arise, and they were addressed on a case-by-case basis. Patients with no clinical follow-up at approximately 1 year were excluded (Fig. 1). Furthermore, patients with incomplete imaging were excluded from analysis at the primary investigators' and study coordinators' discretion if the problem was thought to affect the primary endpoint. For example, the absence of a postoperative brain or cervical spine MRI study, both of which are essential to determine syrinx size and PFD adequacy, led to patient exclusion, while the absence of a postoperative lumbar MRI study did not.

\section{Pros and Cons of Tonsillar Reduction}

Advocates of tonsillar reduction consider it a safe procedure that ensures optimal expansion of the foramen magnum and patency of the CSF pathways with a low complication rate..$^{2,42,18,23,31}$ In fact, some surgeons favor tonsillar resection with minimal bony decompression. ${ }^{3,18}$ It is presumed that because chronic compression renders the tonsils atrophic and gliotic, ${ }^{10,20}$ removing them adds no neurological injury. Notably, a histological study by $\mathrm{Pu}-$ eyrredon et al. showed that, of 43 patients who had undergone cerebellar tonsillectomy for CM-I decompression, 38 had evidence of microscopic tonsillar abnormalities, while only 5 had normal tonsils. ${ }^{20}$

Conversely, proponents of duraplasty without tonsillar reduction contend that once the foramen magnum is expanded by the dural opening, the additional benefit of further expansion by reducing brain tissue mass would be negligible. ${ }^{1,9,28}$ In their institutional review of 500 Chiari malformation decompressions, Tubbs et al. relegated tonsillar resection to the most severe cases, which comprised $<10 \%$ of their cohort, with excellent results overall. ${ }^{27}$ Importantly, intradural dissection and tonsillar coagulation may cause long-term arachnoidal adhesions and resultant foramen magnum obstructions.,15,16 For example, Klekamp showed that 31 of 61 patients with recurrent symptoms after Chiari malformation decompression suffered from arachnoidal adhesions, the majority of whom were patients who had undergone tonsillar resection. ${ }^{15}$

Although cerebellar tonsillar reduction is not associated with discernible neurological deficits, it is likely that all brain regions exhibit distinct neurological functions. The cerebellum contains nearly $80 \%$ of all intracranial neurons, ${ }^{13}$ which implies that it is likely to play a vital functional role, possibly as a coordinating center. Future advances in diagnostic technology may allow subtle neurological sequelae of tonsillar injury to be detected. Still, in the absence of longitudinal studies with large samples that show the procedures' comparative effectiveness, controversies remain.

TABLE 7. Sample size for future studies

\begin{tabular}{ccccc}
\hline \multicolumn{2}{c}{ Percentage } & Improvement & & \\
\cline { 1 - 2 } Control Group & Treatment Group & Risk Ratio & No. Needed per Group \\
\hline $52 \%$ & $64 \%$ & 1.23 & 265 \\
\hline $52 \%$ & $68 \%$ & 1.31 & 146 \\
\hline $52 \%$ & $78 \%$ & 1.50 & 52 \\
\hline
\end{tabular}

This table displays the sample size needed to show relative risk given a $52 \%$ risk in a control group for $80 \%$ power and $\alpha=0.05$. 


\section{Other Surgical Techniques}

The present study was predicated on the assumption that duraplasty is necessary for optimal surgical outcome and, accordingly, did not address the technique of boneonly decompression, which has become more popular in the past several years. Ongoing research that compares bone-only PFD and PFDD duraplasty will be an important adjunct to this. It also might be sensible to assume that specific surgical techniques may be more suitable for patients with specific clinical or imaging characteristics, in which case, large patient registries that include all 3 approaches along with technical variations (e.g., PFDD with or without arachnoid opening and fourth ventricular exploration) would be necessary to categorize surgical indications based on these criteria.

\section{Conclusions}

This pilot study identified no discernible impact on imaging or clinical 1-year outcomes when cerebellar tonsillar reduction was added to bony decompression and duraplasty in the treatment of CM-I with syringomyelia. Additionally, this study sets the stage for future large, prospective studies and registries that compare surgical treatments for CM-I with associated syringomyelia, and it provides data to power a study that can conclusively establish the role, if any, of tonsillar reduction in achieving significant syrinx improvement after CM-I decompression.

\section{Acknowledgments}

The ASAP provided the majority of funding for the study. The Clinical and Translational Science Award program provided funding for statistical analyses through the NIH National Center for Advancing Translational Sciences (grant UL1TR002373). We alone are responsible for the content, and it does not necessarily represent the NIH's official views.

We would like to thank the Office of Clinical Trials of the staff at the University of Wisconsin Institute for Clinical and Translational Research, in particular Connie Trantow, for help in coordinating the study, budgeting, and regulatory services, as well as the staff at the University of Wisconsin Carbone Cancer Center (UWCCC) Biostatistics Shared Resource for their valuable contributions to this research. Shared research services at the UWCCC are supported by a Cancer Center Support grant (P30 CA014520).

This study is dedicated to the memory of Marcy Speer, who was its original driving force and architect.

\section{References}

1. Asgari S, Engelhorn T, Bschor M, Sandalcioglu IE, Stolke D: Surgical prognosis in hindbrain related syringomyelia. Acta Neurol Scand 107:12-21, 2003

2. Bao CS, Liu L, Wang B, Xia XG, Gu YJ, Li DJ, et al: Craniocervical decompression with duraplasty and cerebellar tonsillectomy as treatment for Chiari malformation-I complicated with syringomyelia. Genet Mol Res 14:952-960, 2015

3. Beecher JS, Liu Y, Qi X, Bolognese PA: Minimally invasive subpial tonsillectomy for Chiari I decompression. Acta Neurochir (Wien) 158:1807-1811, 2016

4. Bertrand G: Chapter 26. Dynamic factors in the evolution of syringomyelia and syringobulbia. Clin Neurosurg 20:322333, 1973

5. Chotai S, Medhkour A: Surgical outcomes after posterior fossa decompression with and without duraplasty in Chiari malformation-I. Clin Neurol Neurosurg 125:182-188, 2014
6. Donoghoe MW, Marschner IC: logbin: An R package for relative risk regression using the log-binomial model. J Stat Softw 86:1-22, 2018

7. Durham SR, Fjeld-Olenec K: Comparison of posterior fossa decompression with and without duraplasty for the surgical treatment of Chiari malformation Type I in pediatric patients: a meta-analysis. J Neurosurg Pediatr 2:42-49, 2008

8. Ellenbogen RG, Armonda RA, Shaw DW, Winn HR: Toward a rational treatment of Chiari I malformation and syringomyelia. Neurosurg Focus 8(3):E6, 2000

9. Fischer EG: Posterior fossa decompression for Chiari I deformity, including resection of the cerebellar tonsils. Childs Nerv Syst 11:625-629, 1995

10. Friede RL, Roessmann U: Chronic tonsillar herniation: an attempt at classifying chronic hernitations at the foramen magnum. Acta Neuropathol 34:219-235, 1976

11. Greenlee JD, Donovan KA, Hasan DM, Menezes AH: Chiari I malformation in the very young child: the spectrum of presentations and experience in 31 children under age 6 years. Pediatrics 110:1212-1219, 2002

12. Guyotat J, Bret P, Jouanneau E, Ricci AC, Lapras C: Syringomyelia associated with type I Chiari malformation. A 21-year retrospective study on 75 cases treated by foramen magnum decompression with a special emphasis on the value of tonsils resection. Acta Neurochir (Wien) 140:745-754, 1998

13. Herculano-Houzel S: The human brain in numbers: a linearly scaled-up primate brain. Front Hum Neurosci 3:31, 2009

14. Kennedy BC, Nelp TB, Kelly KM, Phan MQ, Bruce SS, McDowell MM, et al: Delayed resolution of syrinx after posterior fossa decompression without dural opening in children with Chiari malformation Type I. J Neurosurg Pediatr 16:599-606, 2015

15. Klekamp J: Neurological deterioration after foramen magnum decompression for Chiari malformation type I: old or new pathology? J Neurosurg Pediatr 10:538-547, 2012

16. Klekamp J, Batzdorf U, Samii M, Bothe HW: The surgical treatment of Chiari I malformation. Acta Neurochir (Wien) 138:788-801, 1996

17. Krishna V, McLawhorn M, Kosnik-Infinger L, Patel S: High long-term symptomatic recurrence rates after Chiari-1 decompression without dural opening: a single center experience. Clin Neurol Neurosurg 118:53-58, 2014

18. Lazareff JA, Galarza M, Gravori T, Spinks TJ: Tonsillectomy without craniectomy for the management of infantile Chiari I malformation. J Neurosurg 97:1018-1022, 2002

19. Milhorat TH, Chou MW, Trinidad EM, Kula RW, Mandell M, Wolpert C, et al: Chiari I malformation redefined: clinical and radiographic findings for 364 symptomatic patients. Neurosurgery 44:1005-1017, 1999

20. Pueyrredon F, Spaho N, Arroyave I, Vinters H, Lazareff J: Histological findings in cerebellar tonsils of patients with Chiari type I malformation. Childs Nerv Syst 23:427-429, 2007

21. Rocque BG, George TM, Kestle J, Iskandar BJ: Treatment practices for Chiari malformation type I with syringomyelia: results of a survey of the American Society of Pediatric Neurosurgeons. J Neurosurg Pediatr 8:430-437, 2011

22. Sakaki T, Tsunoda S, Morimoto T, Utsumi S: Is Chiari I malformation in the aged initiated by mechanical factors? Report of three cases. Neurol Med Chir (Tokyo) 30:324-328, 1990

23. Stanko KM, Lee YM, Rios J, Wu A, Sobrinho GW, Weingart $\mathrm{JD}$, et al: Improvement of syrinx resolution after tonsillar cautery in pediatric patients with Chiari Type I malformation. J Neurosurg Pediatr 17:174-181, 2016

24. Strahle J, Muraszko KM, Kapurch J, Bapuraj JR, Garton HJ, Maher CO: Chiari malformation Type I and syrinx in children undergoing magnetic resonance imaging. J Neurosurg Pediatr 8:205-213, 2011

25. Strahle J, Smith BW, Martinez M, Bapuraj JR, Muraszko 
KM, Garton HJ, et al: The association between Chiari malformation Type I, spinal syrinx, and scoliosis. J Neurosurg Pediatr 15:607-611, 2015

26. Tubbs RS: Definitions and anatomic considerations in Chiari I malformation and associated syringomyelia. Neurosurg Clin N Am 26:487-493, 2015

27. Tubbs RS, Beckman J, Naftel RP, Chern JJ, Wellons JC III, Rozzelle CJ, et al: Institutional experience with 500 cases of surgically treated pediatric Chiari malformation Type I. J Neurosurg Pediatr 7:248-256, 2011

28. Vanaclocha V, Saiz-Sapena N, Garcia-Casasola MC: Surgical technique for cranio-cervical decompression in syringomyelia associated with Chiari type I malformation. Acta Neurochir (Wien) 139:529-540, 1997

29. Welch K, Shillito J, Strand R, Fischer EG, Winston KR: Chiari I "malformations" - an acquired disorder? J Neurosurg 55:604-609, 1981

30. Wetjen NM, Heiss JD, Oldfield EH: Time course of syringomyelia resolution following decompression of Chiari malformation Type I. J Neurosurg Pediatr 1:118-123, 2008

31. Williams B: Surgery for hindbrain related syringomyelia. Adv Tech Stand Neurosurg 20:107-164, 1993

32. Woodward M: Epidemiology Study Design and Data Analysis. Boca Raton: Chapman \& Hall/CRC, 2005

33. Wu T, Zhu Z, Jiang J, Zheng X, Sun X, Qian B, et al: Syrinx resolution after posterior fossa decompression in patients with scoliosis secondary to Chiari malformation type I. Eur Spine J 21:1143-1150, 2012

\section{Disclosures}

The authors report no conflict of interest concerning the materi- als or methods used in this study or the findings specified in this paper.

\section{Author Contributions}

Conception and design: Iskandar, George, Kestle. Acquisition of data: Koueik, Sandoval-Garcia, Rocque, Haughton, Rebsamen. Analysis and interpretation of data: Koueik, Sandoval-Garcia, Rocque, Rajamanickam, Broman, Iskandar. Drafting the article: Iskandar, Koueik, Sandoval-Garcia. Critically revising the article: Iskandar, Koueik, Sandoval-Garcia, George, Kestle, Rocque, Pollack, Keating, Tuite, Tubbs. Reviewed submitted version of manuscript: Iskandar, Koueik, Sandoval-Garcia, Kestle, Rocque, Frim, Grant, Keating, Muh, Oakes, Pollack, Selden, Tubbs,

Tuite, Warf, George. Statistical analysis: Kestle, Rajamanickam, Broman. Study supervision: Iskandar, George.

\section{Supplemental Information}

\section{Previous Presentations}

Preliminary results of the study were the subject of a podium presentation at the 44th Annual Meeting of the AANS/CNS Section on Pediatric Neurological Surgery, December 7, 2016, Orlando, Florida.

\section{Correspondence}

Bermans J. Iskandar: University of Wisconsin Hospitals and Clinics, Madison, WI. iskandar@neurosurgery.wisc.edu. 